Int. J. Dev. Biol. 50: 157-168 (2006)

doi: $10.1387 / \mathrm{ijdb} .052059 \mathrm{ec}$

\title{
Geometry and mechanics of teleost gastrulation and the formation of primary embryonic axes
}

\author{
ELENA M. CHERDANTSEVA and VLADIMIR G. CHERDANTSEV* \\ Department of Biological Evolution, Faculty of Biology, Moscow State University, Moscow, Russia
}

\begin{abstract}
Examination of normal shaping dynamics and immediate and long-term responses to blastoderm cutting in zebrafish and loach embryos prior to the onset of gastrulation and during the course of epiboly revealed that anteroposterior (AP) and dorsoventral (DV) polarity formation is connected with shaping of the blastoderm circumferential region, which stretches along and shrinks across its movement axes and originates the non-isotropic fields of tensile stresses. Based on data from cutting experiments and quantitative morphology, we reconstructed the movementshaping patterns of epiboly and embryonic shield formation. We revealed that AP and DV axes originate as a mass cell movement subject to the movement-shaping equivalence principle, which means the spatial series of differently shaped areas corresponding to the time succession of the same area shaping. Maintenance of the main body axes in orthogonal orientation depends on the mechanical equilibrium principle allowing for converting shape asymmetry into that of tensile stresses and vice versa. The causal relationship between the main movement-shaping axes and that of embryonic polarity was proved in cutting experiments in which the DV axis direction was subject to rearrangement so as to adjust to the new direction of mass cell movement axes induced by healing the wound in the blastoderm circumferential region.
\end{abstract}

KEY WORDS: morphogenesis, gastrulation, axial pattern, tensile stress, zebrafish, loach

\section{Introduction}

It is widely accepted that the primary embryonic axes, the anterior-posterior (AP) and dorsal-ventral (DV) axes of the embryo, afford a coordinate system for embryonic cells to supply each cell, a cell group, or even each fragment of a cell body by a referent position that unambiguously determines their fate (Weismann, 1893: Wolpert, 1996; however, contrast Gordon, 1999; Cherdantsev, 2003). It was not a matter of chance that the first objection against Turing's model of self-organization in the embryonic systems devoid of prior referent axes (Turing, 1952) was that such axes always exist in normal embryogenesis, their existence preceding the structure that develops (Waddington, 1965).

However, this is not always the case. There are many cases in which the embryonic axial patterns originate on the basis of selforganization, that is, on the basis of a random choice both of the orientation of embryonic axes and disposition of their poles, that the random choice of a place at which a given structure is to be formed can be treated as a common morphogenetic phenomenon. The choice of DV axis direction in embryos with equal spiral cleavage (Arnolds et. al., 1983), or AP axis direction in normal development of hydroids with irregular cleavage (Cherdantsev, Krauss, 1996; Krauss, Cherdantsev, 1999), provide carefully studied examples.

The heuristic alternative view might consist in the inversion of the usually accepted causal relationship between the axial pattern of the embryonic morphogenesis and morphogenesis itself. Instead of considering the movement vectors of material elements, which can be cells, cell groups or cell body fragments, as being dependent on a position of these particles in the special coordinate system imposed on the embryo, we may assume that the main body axes arise as a result of the collective movement of these elements, providing that on a macroscopic scale their collective movement has an axial pattern on its own. For instance, when we are describing the main gastrulation movement in Chordates, we can say that the cells move from the anterior to posterior and from the ventral to dorsal poles of AP and DV gastrula axes - the common description used in text-books. This mode of connection (not interaction!) between the cell movement

Abbreviations used in this paper: AP, anteroposterior; DV, dorsoventral; ESH, embryonic shield; ER, embryonic ring; CE, cell envelope; YSL, yolk syncitial layer; YC, yolk cell.

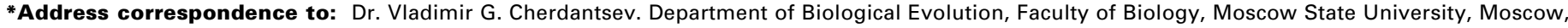
119992 Russia. Fax: +7-495-939-4309. e-mail: arnos@orc.ru
} 
vectors and previously fixed coordinates axes is shown in Fig. 1A. This figure presents the idealized scheme of cell flows in the blastopore marginal zone (blastopore circumference) in Chordates. Note that the increase in the concentration of cells (shown by dots in the figure) in the dorsal centre which, in Amphibians, can be the dorsal blastopore lip, or the embryonic shield in Teleost embryos, is the only morphological effect of DV polarity.

Instead, one might describe the directional mass cell movement in geometrical terms, without reference to any special coordinate system. Each area moves so as to stretch along and shrink across its movement axis. This shapes the marginal zone circumference as shown in Fig. 1B. The convergence of cell flows shown in this figure (arrows) is an inevitable effect of this movement-shaping mode, each circumference sector being a potential dorsal centre of the embryo $\left(D, D^{\prime}\right.$ and $\left.D^{\prime}\right)$. Taking into account the fact (see Cherdantsev, 2003) that there is a positive feedback between shaping this circumference in any given sector and converging the cell flows and a negative feedback between the different centers of convergence, the competition between the potential centers $D, D$ 'and $D$ 'for cell recruitment results in only one centre (presumably centre $D$ in the figure) whose position will determine the direction of the DV axis.

The last scenario outlining the morphodynamics approach to understanding the origin of embryonic axes suggests two problems. One is how the dorsal centre of the gastrula circumference manages to have the same position in the embryo as it occupied in the egg and in the blastula. The principal answer is quite obvious: its position in the gastrula is a matter not of a random, but rather of a biased choice (as in the competition of "problastopores", Gordon, Björklund and Nieuwkoop, 1994). The difference in developmental history of different marginal zone sectors can bestow on one of them the advantage in the competition for recruiting the neighboring cells with the onset of gastrulation. However, it remains open to question what is the nature of this advantage and how the morphogenetic processes of different time and space scales can be matters of the same polar and axial organization of the whole embryo.

A

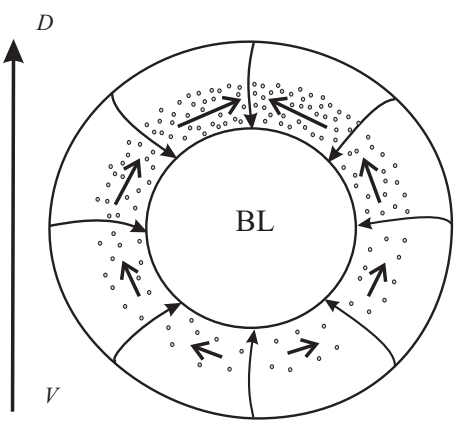

B

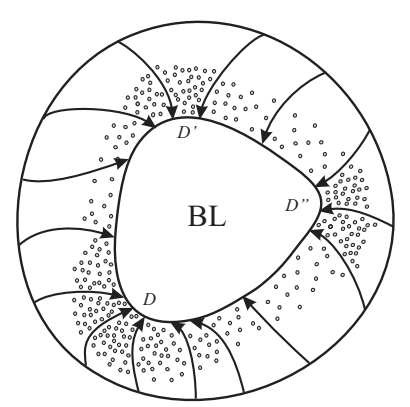

Fig. 1. Scheme illustrating the alternative models of the acquisition of DV asymmetry in the generalized gastrula of chordates. (A) Coordinate model. (B) Morphodynamics model. BL, blastopore circumference; dots, the concentration of cells in the circumferential region; arrows, mass cell flows; D-D", potential dorsal sectors. The scales of shaping of the blastopore circumference in (B) are exaggerated, in order to stress the difference between the Coordinate and Morphodynamics models.
The other problem is even of a greater importance. If we assume that the formation of the main embryonic axes arises as a result of shaping of the embryo, then why does this shaping emerge on a macroscopic scale only at the beginning or even in the end of gastrulation? The traditional view rests on the assumption, rather than on the observation, that the polar and axial patterns can be arranged or even re-arranged in the embryo whose shape remains that of a perfect sphere. If this were the case, any kind of feedback between the movement of material elements and shaping of both these elements and the whole embryo in which they move, would be irrelevant. The connection between the pattern formation and morphogenesis proper would be as shown in Fig. 1A. On the other hand, if this is not the case, one needs to explain why the shape changes seem not to be explicitly manifested. Note that, in Fig. 1B, to illustrate the morphodynamics alternative to the coordinate model, the scales of shaping the gastrula are exaggerated.

Yet, in this work we are intending to show, taking gastrulation of Teleost embryos as a model, that the seemingly symmetrical shape of the embryo is backed by an asymmetry of the mechanical stresses. The phases of active shaping alternate with phases in which the shape asymmetry turns into mechanical stresses and vice versa. Providing that shaping arises as a result of mass cell movement at the whole embryo scale and that it is imprinted in the asymmetry of mechanical stresses, the mechanical equilibrium principle allows both for the origin of the primary embryonic axes and their direction and positioning remaining unchanged in spite of complicated movement-shaping patterns.

The first part of this work is a review of our work on zebrafish (Danio/Brachidanio rerio) published in Russian (or unpublished). In this part we report morphological and experimental data concerned with DV axis formation. The second part is based on new data obtained for loach embryos. In this part we analyze cell movement geometry and its interaction both with the movement itself and patterns of mechanical stresses being "charged" by cell movement and "discharged" when movement is subject to rearrangement.

\section{Dorsoventral asymmetry in zebrafish}

The early development of Teleosts shows how geometrical embryogenesis can be. Up to the onset of gastrulation the blastoderm cells form a single homogeneous population (Ballard, 1966; 1981). One could say (see Cherdantsev, 2003), that the ancestral heterogeneity of embryonic tissues (in Vertebrates with total cleavage) turns into extra-embryonic regions forming the blastoderm environment, the outer cell envelope (CE), yolk syncitial layer (YSL) and yolk cell (YC) (Betchaku and Trinkaus, 1978).

Yet it has been commonly accepted (see, for example, Kimmel et. al., 1990) that the first reliable morphological signs of DV polarity arise only with formation of the embryonic shield (ESH), the morphogenetic analogue of the dorsal blastopore lip in Amphibian embryos. This is not the case (Cherdantseva, Cherdantsev, 1985; Schmitz, Campos-Ortega, 1994). At the very beginning of gastrulation the shape of zebrafish embryos both in optical and histological sagittal sections, as shown in Fig. 2A, is devoid of radial symmetry (the dorsal side is on the left). When looking at the circumferential boundary separating the blastoderm and $Y C$, one can see that in one region of the embryo both the outer blastoderm 
A
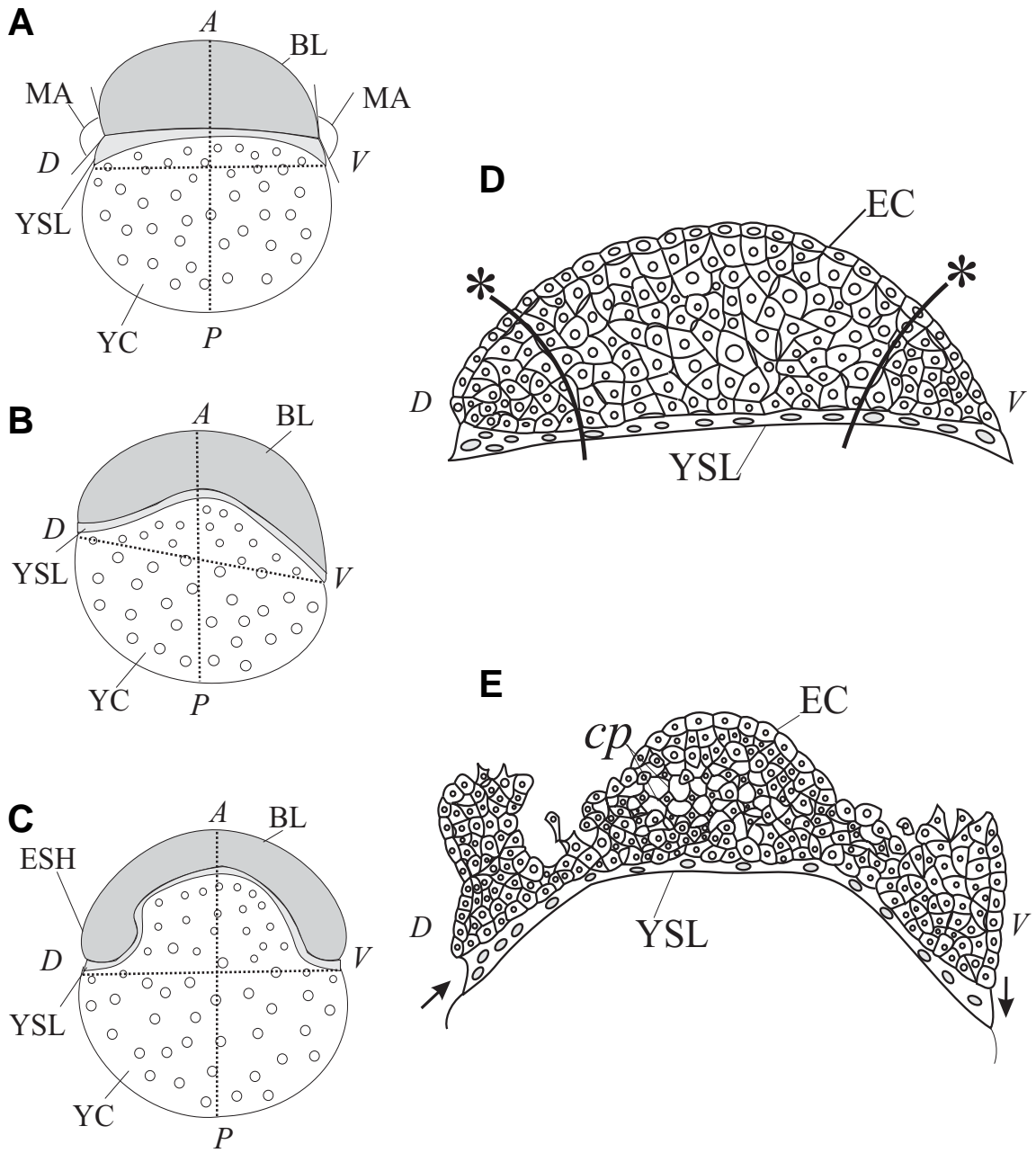

Note, as shown in Fig. 2B, that at the early steps of epiboly, the ER being only in status nascendi, the embryonic axial pattern is distorted. If, in Fig. 2A, the AP axis joining the geometric centers of the blastoderm and the YC is perpendicular to the DV axis joining opposite blastoderm margins, this is not the case in Fig $2 \mathrm{~B}$, as at the ventral blastoderm margin the degree of epiboly is higher than at the dorsal margin. With completion of ER formation, the orientation of the primary embryonic axes regresses (Fig. 2A) and this is at the expense of $\mathrm{ESH}$ formation, that is, at the expense of concentrating ER cells in the dorsal ER sector (Fig. 2C).

Fig. 2D shows the shape and arrangement of the blastoderm cells in a sagittal histological section made at the stage shown in Fig. 2A. The asterisks mark the curves constructed so as to cross the outer blastoderm surface and that of the YSL with a minimal deviation from their normal radii of curvature. To a first approximation these curves can be considered as parts of the circumference. If we assume that the epiboly vectors are reciprocally proportional to their radii of curvature (which is reasonable, as the blastoderm surface area increases at the expense of shrinking the blastoderm across the epiboly direction), the difference in radii of curvature is balanced by the decrease in the blastoderm thickness, this permitting both margins to overgrow the $Y C$ at the same rates.

It follows that epiboly geometry has a property of regulating its own dynamics not only in an indirect (parametric, see Beloussov, 1998), but also in a direct way (Cherdantsev, 2003). When we cut the blastoderm by two incisions made across the DV axis at equal distances from the dorsal and ventral blastoderm poles (Cherdantseva, Cherdantsev, 1985), the wound Fig. 2. Shaping succession, histological structure and tensile stresses in the epiboly of zebrafish embryos. (A-C) Successive shapes of the whole embryo during the normal epiboly. (D) Histological structure of the intact blastoderm at the onset of epiboly. (E) Its immediate rearrangement after cutting. $D$, dorsal; $V$, ventral side; $A$, anterior; $P$, posterior pole; modified from Cherdantsev, 2003; for other explanations, see text.

surface and that of $Y C$ are inscribed in a single spherical surface, which means the marginal angle (MA) at their separation boundary tends to be equal to $180^{\circ}$. In the opposite region these surfaces have a different curvature, the MA being always less than $180^{\circ}$ (see Fig. $2 \mathrm{~A}$ ). These observations and marking experiments show that it is this region that becomes the dorsal side of the embryo (Cherdantseva and Cherdantsev, 1985; confirmed by Schmitz, Campos-Ortega, 1994).

Gastrulation starts when the initially flat surface separating the blastoderm and $Y C$ acquires a convex shape. The blastoderm starts to increase its surface area on the expense of decreasing its thickness. The blastoderm, YSL and CE begin to overgrow YC surface. It is this movement, involving both the embryonic and extra-embryonic tissues, that is usually called epiboly, but, to escape ambiguity, we are referring it only to the movement of blastoderm cells. As the increase in the blastoderm surface area implies that the mass movement of blastoderm cells is towards the blastoderm circumference (centrifugal cell migration, see Ballard, 1981), it forms the blastoderm embryonic ring (ER). margins immediately break apart. In a minute or less the embryo acquires a shape shown in Fig. 2E. Note that the shape changes in the dorsal and ventral blastoderm margins are in opposite directions, shown by arrows, which means that the marginal point of the dorsal blastoderm "lip" is subject to centripetal shift, while the ventral "lip" is subject to centrifugal shift. As these changes occur almost immediately after cutting, they can be referred to the relaxation of tensile stresses accumulated both in embryonic and extra-embryonic tissues.

The relaxation of tensile stresses (cf. Figs. 2D and E) makes it clear that the blastoderm, before the onset of gastrulation, represents a tensile cell network, the cells being connected with neighboring cells, CE and YSL by cell surface processes. It is easy to see that such a network imposes no constraints on a cell changing its neighbors. The elongated processes are not prominent in histological section of the intact blastoderm simply because they tend to shorten. This provides a potent source of tensile forces arising with the mass cell movement.

A diagram of the tensile cell network is shown in Fig. 3A (the 

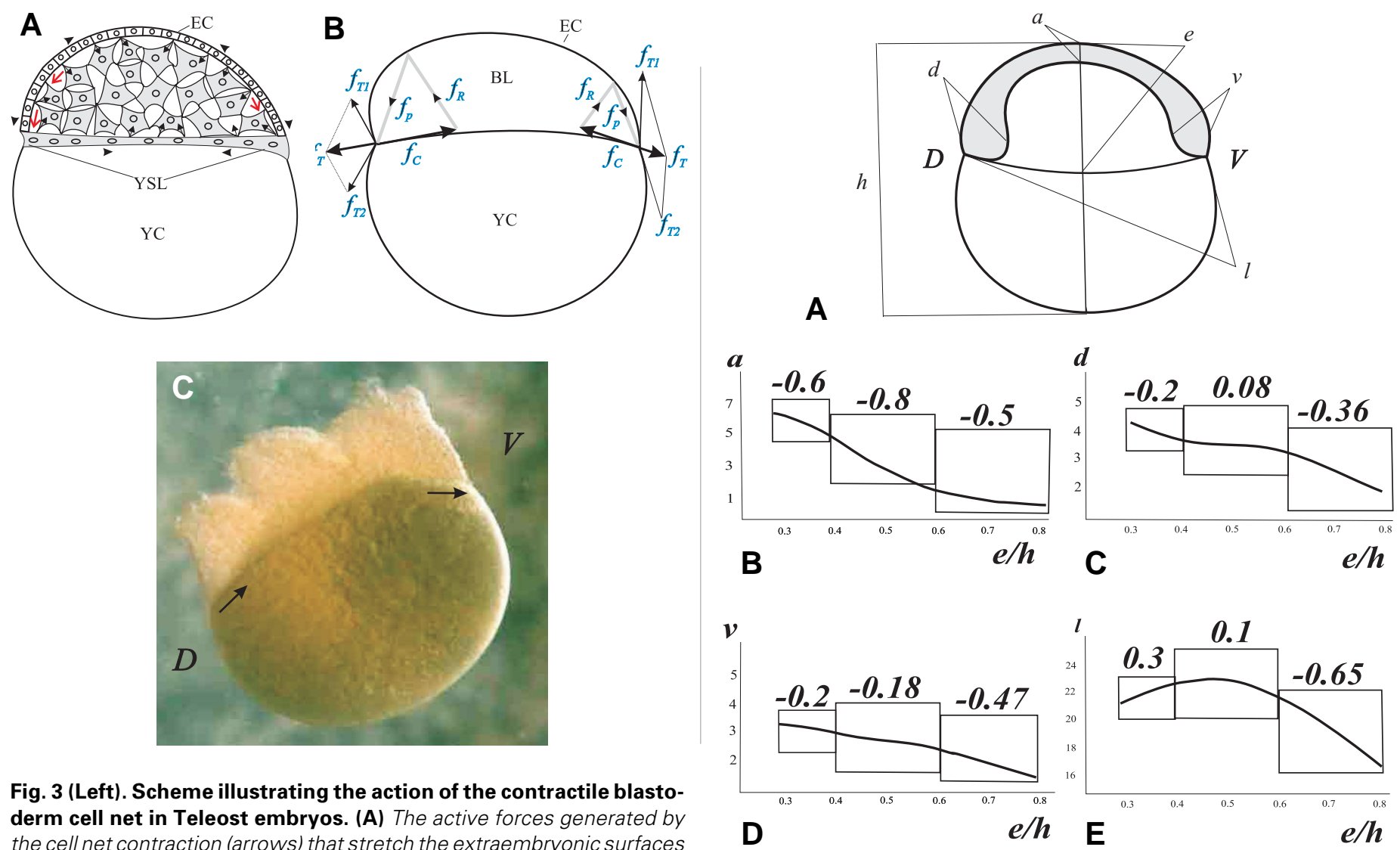

Fig. 3 (Left). Scheme illustrating the action of the contractile blastoderm cell net in Teleost embryos. (A) The active forces generated by the cell net contraction (arrows) that stretch the extraembryonic surfaces (arrowheads). Red arrows indicate cell movement vectors. (B) The pattern of tensile forces; for their designation and explanation see text. (C) The illustration, via the optic section of a living embryo, of the immediate response of the loach embryo to cutting (for explanation, see text). Arrows, the directions of a shift in the positions of the blastoderm margins; $D$, dorsal; $V$, ventral side.

Fig. 4 (Right). Quantitative dynamics of morphological characters in the epiboly of loach embryos. (A) Scheme of measurements. (B-E) The dependences of measured morphological characters (ordinates) on the relative degree of epiboly (abscissa). Shown above the rectangles are the coefficients of correlation between the ordinates and abscissa in the outlined abscissa intervals.

dorsal side is on the left). For simplicity it shows only a few blastoderm cells, each being connected by processes both with neighboring cells and CE and YSL surfaces. Contraction of the cell processes, shown in Fig. 3A by arrows, stretches both the CE and YSL surfaces (arrowheads). Then (Fig. 3B), at the intersection point between these surfaces and the blastoderm-YC boundary, the $C E$ and $Y C$ surfaces are under stretch (the forces $f_{T 1}$ and $f_{T 2}$ in Fig. 3B) arising as a result of tensile reaction to the active contraction of the blastoderm network (to the force $f_{C}$ in Fig. 3B). As follows from the experiment shown in Figs. $2 \mathrm{E}, \mathrm{F}$ and other cutting experiments (see Cherdantseva, Cherdantsev, 1985), the dorsal blastoderm "lip" exceeds the ventral "lip" in contractile activity. This activity is greater than one could have drawn from a difference in the MA values shown in Fig. 2A. Then, to get a mechanical equilibrium between the dorsal and ventral sides, one needs, as shown in Fig. 3B, to take into account the intrinsic pressure (the force $f_{p}$; see red arrows in Fig. 3A) which is exerted by cells migrating towards the blastoderm circumference and adds to the blastoderm margin stretching preponderantly on the dorsal side. This addition compensates for the preponderance of the force $f_{C}$ (the force $f_{R}$ shown in Fig. 3B, is a counterbalance of vector sum of $f_{C}$ and $f_{p}$ ).
It follows that DV polarity in zebrafish embryos is a matter of equilibrium between the pressure which Bollard's centrifugal cell migration (Bollard, 1981) exerts on the blastoderm circumference and the blastoderm cell network contractile activity, to which is presumably added that of the YSL - a multinuclear cell possessing its own network of contractile filaments (Trinkaus, 1993).

The distinctive features of the morphodynamics model can be summarized as follows. First, polarization depends not on material gradients, but rather on the mass movement-shaping of embryonic regions. Second, axial organization is subject to a mechanical equilibrium principle allowing for the orthogonal orientation of movement-shaping vectors at the scale of the whole embryo. Third, the same polarization mechanism can be reiterated on smaller and smaller scales, such as in the formation of the $\mathrm{ESH}$, which we now consider.

\section{Epiboly morphodynamics and embryonic shield for- mation in loach embryos}

\section{The quantitative morphology of epiboly}

In loach (Missgurnus fossilis L.) embryos DV polarity is distinguishable prior to the onset of gastrulation on the basis of the 
same geometric differences found in zebrafish (Nekliudova et. al., 2000; Cherdantsev, Tsvetkova 2005). The immediate response to transversal cuts made at similar distances from the dorsal and ventral blastoderm margins is fairly similar to zebrafish (Fig. 3C, cf. Fig. 2E), suggesting the same pattern of operating forces, shown in Fig. 3B.

The quantitative data on the course of epiboly were obtained on the egg clutches fertilized in the lab after hormonal stimulation of females and males. Eggs were reared at room temperature $\left(19^{\circ} \mathrm{C}\right)$. The quantitative morphological characters that roughly approximate the normal morphodynamics of epiboly, are shown in Fig. 4A. These were measured in vivoin each individual embryo at equal time intervals (35 min) using optic sagittal sections of living embryos. Each embryo was measured from the beginning of epiboly, when the blastoderm covers one third of the egg surface and up to ESH segregation, when the blastoderm covers two thirds (or a little more) of the egg surface. The variability of individual developmental paths, which itself merits consideration, is reporting elsewhere (Cherdantsev and Tsvetkova, 2005). Here we consider only an averaged sample of embryos (two hundred embryos from $15 \mathrm{egg}$ clutches). Since the rate of development varies between individual embryos, we take the relative degree of epiboly, that is, the distance between the geometric blastoderm centre and blastoderm circumference $(e)$ divided on the egg height $(h)$ (see Fig. 4A), as the developmental time. The values of conventionally dependent characters (ordinates) are expressed in units equal to $0.05 \mathrm{~mm}$.

Figs. 4B-E show how the morphological characters depend on the relative degree of epiboly. Each curve, shown in these figures, is calculated by the method of least squares and is supplemented by the correlation coefficients $(\lambda)$ between the abscissa $(e / h$, the relative degree of epiboly) and ordinates ( $a, d$, vand $\hbar$ calculated at intervals shown in the graphics.

The thickness of the blastoderm region ( $a$ in Fig. 4B) decreases, the maximum slope being $0.4-0.6$, in $e / h$ proportion. This reflects the decreasing number of blastoderm cell layers and increasing blastoderm surface area, which is known as one of the driving forces both of epiboly and centrifugal cell migration (Ballard, 1981; Kimmel et. al., 1990).

As for the thicknesses of dorsal and ventral blastoderm margins ( $d$ in Fig. 4C and $v$ in Fig. 4D), they decrease only at the beginning and end of epiboly, that is, before and after 0.4-0.6 e/ $\mathrm{h}$ proportion interval. Inside this interval the average thickness does not change and so is the thickness difference between the dorsal and ventral blastoderm margins, the dorsal margin thickness remaining always at a higher level $(p<0.001)$. Thus, when the blastoderm circumference passes through the equatorial region, it is the constancy of thickness difference between the opposite blastoderm margins that allows for maintenance of the orthogonal orientation of the main body axes.

Epiboly is usually portrayed in such a way that the blastoderm seems to spread over the spherical YC surface. If this were the case, the blastoderm would behaved like a drop of a liquid flowing over a solid spherical surface. Then, one could expect a parabolic dependence of the diameter ( $x$ of the blastoderm circumference on the relative degree of epiboly $(e / h)$. However, the empirical dependence, shown in Fig. 4E, is not parabolic one, as in the interval $0.4-0.6$ of the e/h proportion, the slope is close to zero.

It follows that, when the blastoderm approximates the egg equator, its circumference becomes subject to active shaping. At each new step of epiboly the blastoderm circumference tends to reduce the egg's latitudinal diameter so as to equalize it to that at the previous step of epiboly.

\section{Movement-shaping and dorsoventral asymmetry of the blas- toderm circumference when passing the equatorial region}

It follows from the quantitative morphological data that passing through the egg equatorial region (the interval 0.4-0.6 of the relative degree of epiboly) is of crucial importance for further developmental. The point is that, when entering this region, the epiboly movement becomes movement-shaping, while keeping the thickness difference between the dorsal and ventral blastoderm margins constant.

The morphological sign that epiboly is entering the equatorial region is $E R$ formation. Figs. $5 A-C$ show the succession of $E R$ formation via optical sagittal sections of living embryos. Drawn on the left are the normal radii of curvature of the outer blastoderm surface; drawn on the right are the angles between the adjacent embryonic and extra-embryonic surfaces at their intersection points, that is, at the blastoderm margins.

At the onset of ER formation (Fig. 5A) the outer blastoderm surface is perfectly inscribed in a circle having its centre just inside the whole embryo, as it follows from the geometric construction
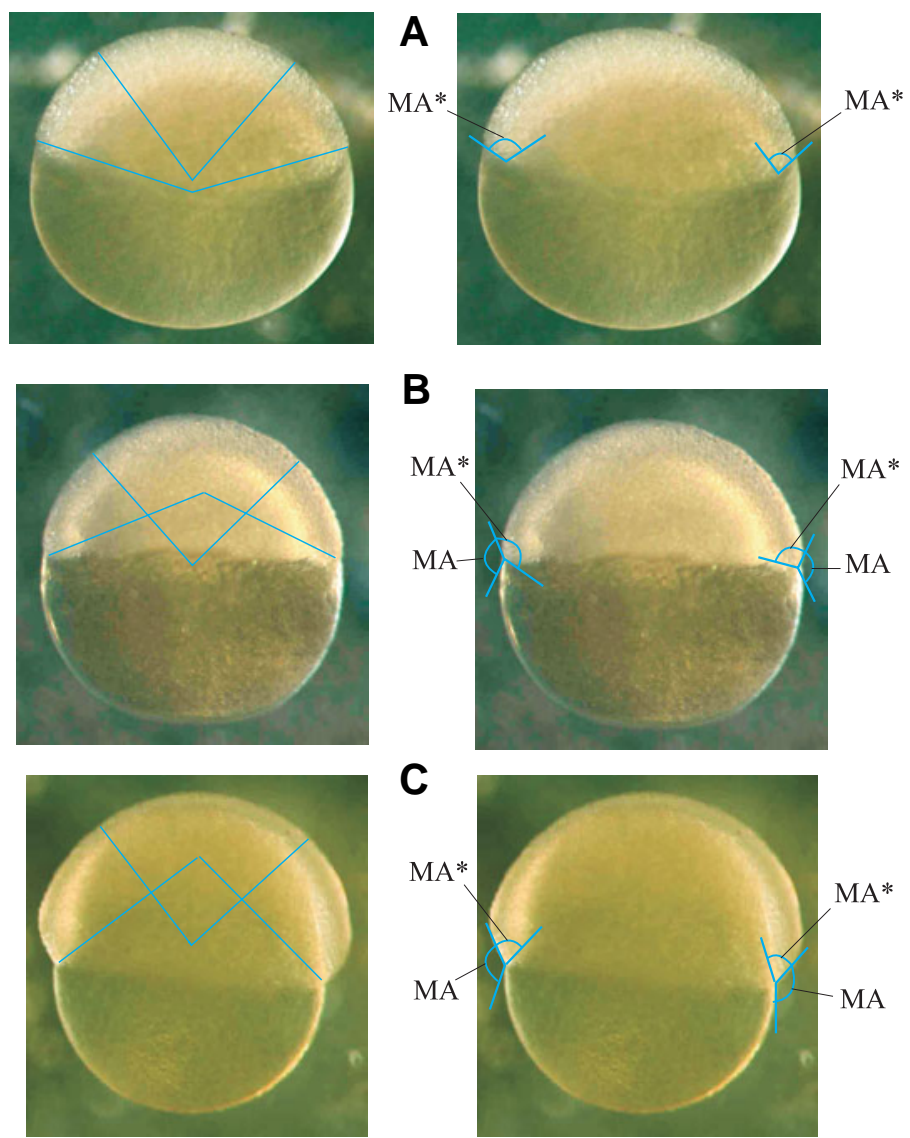

Fig. 5. The succession of epiboly in loach via optical sections of living embryos. (A) Onset of epiboly. (B) Bulging phase. (C) Flattening phase. The dorsal side is on the left; shown on the left column are the radii of curvature of the outer blastoderm surface. 
shown on the left of Fig. 5A. Use of the MA, the angle between the outer blastoderm surface and the $Y C$, which before the onset of epiboly characterized DV asymmetry, becomes inappropriate, if only because it is equal to $180^{\circ}$ throughout the blastoderm circumference, as shown in Fig. 5A on the right. Instead, DV asymmetry is expressed in the shape of the inner blastoderm surface. The angle $\mathrm{MA}^{*}$ between the inner and outer blastoderm surfaces, shown in Fig. 5A on the right, is greater at the dorsal side of the embryo. While MA and MA* are different and geometrically independent morphological characters, their contribution in maintaining DV asymmetry is obviously the same, both indicating that the active surface tension at the separation boundary between the blastoderm and the YC is greater on the dorsal side. When this initially flat separation boundary turns into a convex boundary at the onset of epiboly, its active surface tension decreases, while the difference in $\mathrm{MA}^{*}$ values means that this decrease is delayed at the dorsal side.

With ER formation, the active tension of the blastoderm-YC separation boundary is again increases, but only inside the ER. The blastoderm circumference becomes subject to latitudinal contraction and so the outer embryonic surface is no longer inscribed in a single circle. This follows from the geometric construction shown on the left of Fig. 5B: in the ER region the curvature of the blastoderm outer surface increases as compared

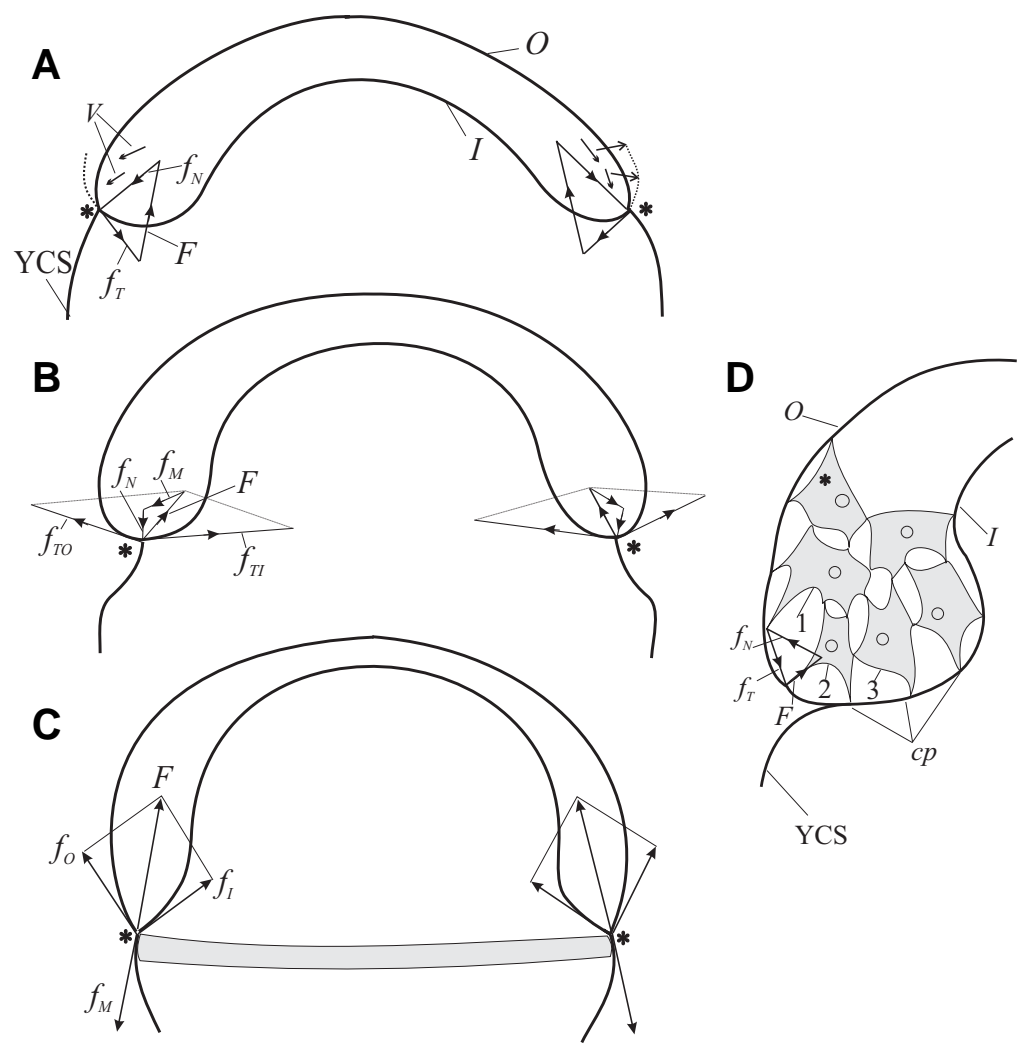

Fig. 6. Dynamic geometry and mechanics of epiboly in loach embryos. (A-C) The bulging-flattening transition: $O$, outer; I, inner blastoderm surfaces; YCS, yolk cell surface; asterisks, the intersection points between the outer and inner blastoderm surfaces; the dorsal side is on the left. (D) The movement-shaping of the blastoderm cell net (cp, cell processes); for the designation of tensile forces (shown only at the dorsal side) and other explanations, see text. to the rest of outer embryonic surface. The "waistline" arising between the blastoderm and the YC (see Fig. 5B) explains why the blastoderm can spread towards the egg equator with no increase in its latitudinal diameter.

After the "waistline" appears both the dorsal and ventral blastoderm poles become the intersection points between the outer blastoderm surface (CE surface), the blastoderm-YC separation boundary (YSL surface) and the outer YC surface, as shown in Fig. 5B on the right. Thus, there are three angles between these surfaces (one is MA, the other is $M A^{*}$, the third is that between the YSL and the $Y C$ ), each having a dynamics of its own in shaping the blastoderm circumference. The reason to take the angle MA* for further analysis is that it is the only angle whose values at the dorsal and ventral blastoderm poles have been proved to be matters of a statistically significant difference $(p<0.001)$ in the epiboly interval $0.4-0.6$ of the $e / h$ proportion, the dorsal MA* being greater than the ventral one (data not shown).

When comparing the dorsal blastoderm margin shapes shown in Figs. 5B and $\mathrm{C}$, it is easy to see that epiboly consists of two movement-shaping phases. In the first phase the blastoderm margin bulges, $\mathrm{MA}^{*}$ increases with little progress in epiboly. In the next phase it flattens, $M A^{*}$ being subject to little decrease with significant progress in epiboly. The ventral margin shaping proceeds in a similar way with one exception: while its epiboly progress is the same as in the dorsal margin (otherwise the AP and DV axes could not have preserved orthogonal orientation), $\mathrm{MA}^{*}$ is always less than at the dorsal margin, as is the margin thickness.

\section{The movement-shaping algorithm and embryonic shield formation}

By definition (Cherdantsev, 2003), movement-shaping has the property that the shape of a moving area contains the algorithm of its movement, which means that the geometry of a region constructs its own movement vectors.

The alternation of bulging and flattening phases described in the previous section follows the algorithm shown in Fig. 6 (dorsal side is on the left). Cell flows, such as that of Ballard's centrifugal migration (see Ballard, 1966), adjust their directions to the shape of the ER contour that they mold. Since at the onset of ER formation the outer and inner ER surfaces have different curvatures, the cells flows, shown in Fig. 6A by vectors $V$, are directed not to the intersection point between these surfaces, but deviate from this shortest route towards the outer surface, so as to equalize its curvature with that of the inner one. The outer ER surface acquires a new (bulged) contour, shown in Fig. 6A by the dotted line, so as to adjust orientation of cell flows to the normal radii of curvature of the surface which they shape. What is important in this shaping process is that it creates a new movement component that makes the intersection point move centripetally, which allows for "waistline" formation.

Then, in the state of mechanical equilibrium, the intersection point is a matter of equilibrium between the force $f_{T}$ pulling this point in the centripetal direction, the force $f_{N}$ bulging this surface from within and reaction 
force $F$ counterbalancing the joint action of these forces (see Fig. $6 \mathrm{~A})$. The bulging ceases and so does the centripetal movement of the blastoderm margin, as, in the neighborhood of the intersection point, the outer surface attains a curvature approximating that of the inner surface.

At the end of the bulging phase these surfaces are at the symmetrization threshold, as shown in Fig. 6B. The bulging force $f_{N}$ decreases and is replaced by force $f_{M}$ which pulls the blastoderm margin along the YC surface (YCS). Both of these forces are counterbalanced by force $F$ whose components, $f_{T O}$ and $f_{T /}$ are that of the tensile stretch of the outer and inner surfaces, respectively. The bulging-flattening transition occurs when $f_{N}$ is equal to zero and the driving forces of epiboly are at equilibrium, as shown in Fig. 6C. In the flattening phase, the movement of the intersection point is along the YCS (see force $f_{M}$ ) being counterbalanced by stretching the outer and inner ER surfaces.

After the blastoderm circumference has passed by the equatorial region, movement-shaping is over, as epiboly is no longer shaping the surface which it moves along. The movement-shaping mechanism is presumably replaced by turning the blastoderm and/or the YSL periphery into a contractile ring (Betchaku and Trinkaus, 1978), shown in Fig. 6C as a grey band.

A more detailed explication of movement-shaping mechanics is given in Fig. 6D. The blastoderm is again portrayed (cf. Fig. 3A) as a contractile cell network. The movement-shaping model rests on the assumption that, as shown in Fig. 6D, the spatial series of cells 1,2 and 3 with more and more processes $(c p)$, corresponds to the succession of shortening the processes of a single cell moving along the outer surface (CE surface) towards the intersection point with the inner surface (YSL). Then, in a mechanical equilibrium state, the shortening (contraction) of cell 2 processes (force $F$ ) pulls cell 1 along the outer surface (force $f_{7}$ ) by increasing this surface's curvature (force $f_{N}$ ) and, consequently, shortening the distance which cell 1 has to pass so as to reach the blastoderm margin. Then, when a new cell, for example, that marked in Fig. $6 D$ by the asterisk, is included into the ER, there originates a positive feedback between its translocation towards the intersection point and shaping of the ER surface which this cell moves along. The greater is the outer ER surface curvature, the easier is for new cells entering the ER to overtake cells that have been included into the ER just before. The cells become crowded inside the ER, with a good likelihood that cells situated at the very blastoderm edge are pushed into to the deeper blastoderm layers. This fits in well with data obtained from direct observations on individual cell movement tracks (Kimmel et. al., 1990).

\section{Embryonic shield formation}

As follows from the morphological pattern dynamics shown in Figs.6A-C, the movement-shaping of dorsal and ventral blastoderm margins differs only in the scale permitting them to preserve the thickness difference they acquired before the onset of epiboly. However, the initial DV asymmetry becomes subject to the complication of being expressed in ESH formation, which is also subject to a simple geometric algorithm.

The outline of the ESH contour becomes apparent immediately after ER formation, as shown in Fig. 7. Its shape is that of a tongue whose wide edge is at the dorsal blastoderm margin (Fig. 7A, B). Then the ESH stretches along and shrinks across the movement axis. Its lateral boundaries become aligned parallel to one another

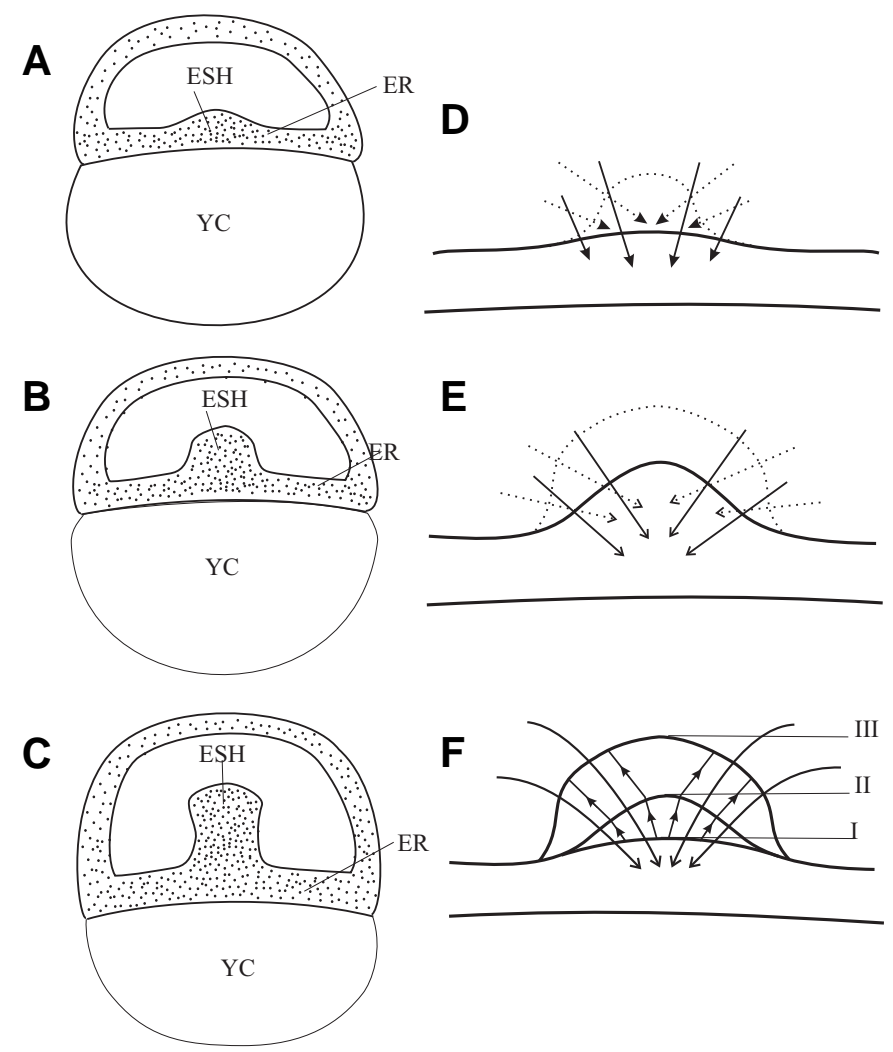

Fig. 7. Movement-shaping of the embryonic shield (ESH). (A-C) Successive shapes of the ESH and embryonic ring (ER) in the loach embryo. (D-F) The movement-shaping algorithm of ESH formation. Solid lines and arrows in $(D, E)$ represent the current. Dotted lines and arrows, the future ESH contours and directions of cell flows; contours I-III in (F), the spatial series of shaping of the contour I (arrowheads); arrows, the cell flows.

(Fig. 7C), this alignment corresponding to the end of the interval 0.4-0.6 of the $e / h$ proportion. The succession of ESH shapes is fairly similar to that of the shaping of the dorsal archenteron roof during amphibian gastrulation (Cherdantsev, 2003).

Note that the description of ESH shaping is geometric, with no mention of the embryonic axes. The connection with their orientation consists only in that ESH is initiated in the dorsal ER sector and its shaping is in the direction of epiboly. The description of its morphogenesis in geometric terms is not simple, insofar as the actual ESH contour may be used to derive its future contour with the help of a geometric construction shown in Figs. 7D, E. To get a future contour from the current one, it is sufficient to construct lines that converge to the ESH initiation centre, their orientation being normal to the ESH contour and assume that these lines are those of mass cell movement tracks. The algorithm consists of two steps. At the first step we construct a new series of lines (the dotted lines in Figs. 7D, E), so as to enhance their convergence. At the next step we describe a new ESH contour (shown by dotted lines in Figs. 7D, E), so as to normalize its orientation with respect to the new configuration of cell movement tracks. We get almost a perfect correspondence between the series of contours being provided by this morphodynamics algorithm and the real succession of ESH contours. The algorithm works only because, as 
A

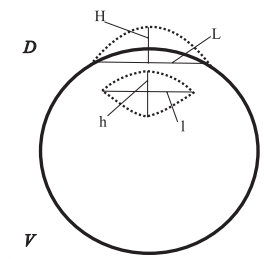

C

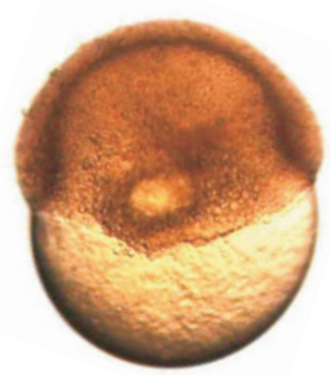

B

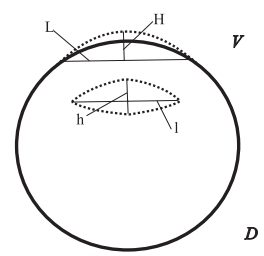

D

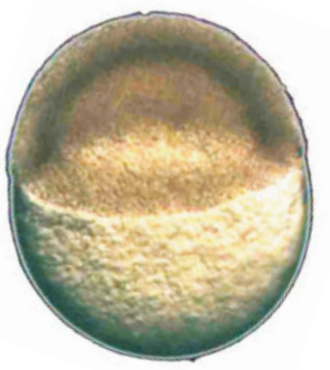

$V$

E

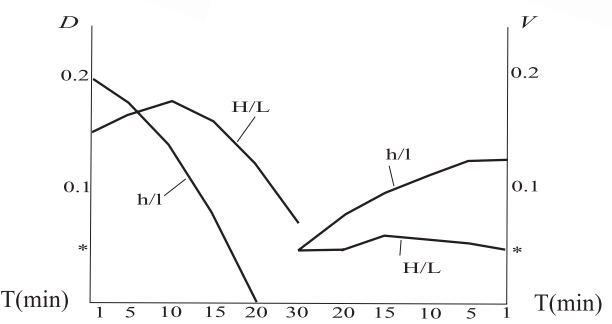

Fig. 8 (Left). Cutting experiments showing the dorsal convergence of tensile stresses. (A,B) Schematic drawings of the immediate response to dorsal (A) and ventral (B) incisions. (C,D) The living embryos after dorsal and ventral incisions.

I) and blastoderm (H/L) circumference shaping at the dorsal (D) and ventral (V) blastoderm margins.

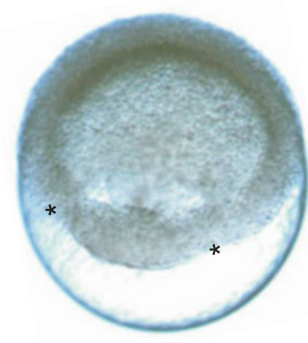

A

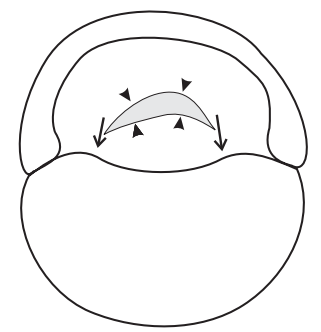

B
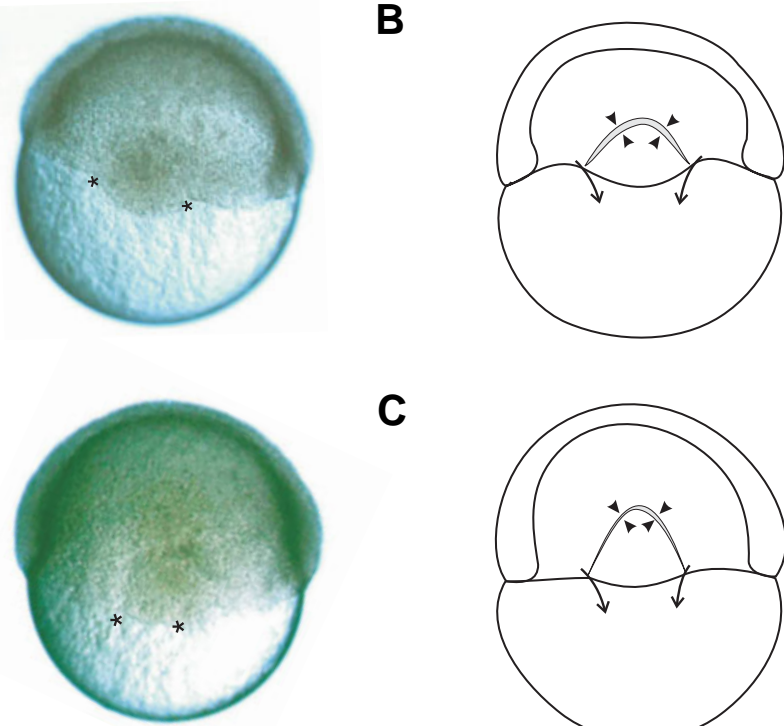

C

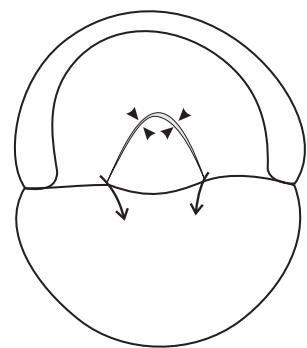

Fig. 9 (Right). Wound healing convergence after dorsal incisions. (A-C) On the left are the dorsal side views of living embryos. The asterisks show interceptions between the wound seams and blastoderm circumference. On the right are the schemes of the cell flows; the arrowheads show the wound healing, the arrows show the blastoderm circumference movement.

shown in Fig. 7F, the spatial series of contours I-III corresponds to the time succession of the shaping of the same contour.

\section{Dorsoventral pattern of tensile stresses accompanying and guiding the mass cell movement}

In this section we report the results of cutting experiments which permit us, first, to understand how the mechanics keeps the AP and DV axes in orthogonal orientation and, second, to visualize the convergence of cell flows.

In the first series of experiments we cut the blastoderm across the DV axis at the dorsal, or at the ventral blastoderm side. The dorsal and ventral incisions were made at equal distances from the dorsal and ventral blastoderm edges, as shown in Fig. 8A (dorsal incision) and B (ventral incision). We made them so as to dissect all the blastoderm layers and to denude the YSL surface. At one minute after the incision was made the live embryo was measured in a manner shown in Fig. 8A, B and then left for further observations and measurements.

Fig. $8 C$ and $D$ show typical images of embryos immediately after the dorsal $(C)$ and ventral (D) incisions. Note that the proportion of the longitudinal-to-latitudinal wound area lengths is greater on the dorsal blastoderm side and so is the change in the blastoderm circumference radius of curvature in the incision sector.

This is confirmed by quantitative data summarized in Fig. 8E, the dorsal side on the left and the ventral side on the right. To decrease the distortion arising from projecting the three-dimensional blastoderm onto the optic plane, we tried to keep the embryos under measurement in a standard orientation: the geometric blastoderm centre upwards. The ordinates in Fig. 8E represents the longitudinal-to-latitudinal ratio of the wound area arising as a result of breaking up the incision margins $(h / h)$ and, as a measure the blastoderm circumference curvature, the heightto-length ratio of the circumference arc in the incision sector $(H)$ $\mathrm{L}$, the scheme of measuring see in Figs. 8A, B). For $h / /$ the initial points are the immediate blastoderm reactions being recorded after one minute; the following points portray wound healing dynamics. For $H / L$ the initial points are the height-to-length ratio of the blastoderm circumference in the incision sectors just before cutting; the subsequent points indicating how the local increase in the curvature of the blastoderm circumference changes after the incision was made. The immediate increase in the local curvature 
is statistically significant only on the dorsal side $(p<0.001)$.

It follows from these graphics that the proportion of longitudinal tensile stress is higher at the dorsal blastoderm margin $(p<0.001)$ and so are the wound-healing rates higher, which indicate the level of blastoderm net contractile activity. Taking into account the fact that the blastoderm circumference is close to that of a circle, we can explain this difference in tensile patterns only provided that both the tensile stress lines and cell flows converge to the dorsal sector of ER. If longitudinal and latitudinal stresses had orthogonal orientation, it would have been unexpected for the latitudinal cut to cause latitudinal shrinkage of the dorsal blastoderm margin in the incision sector. It is this shrinkage that allows both for local increase in the curvature of the blastoderm circumference at the dorsal side and the relatively high longitudinal-tolatitudinal ratio of the wound area.

The wound healing process permits visualization of the convergence of cell flows to the dorsal blastoderm margin occurring in the normal course of epiboly

Healing the wound both after the dorsal and ventral incisions leaves a prominent seam at the incision line which is visible in living embryos for at least a few hours after the operation. The succession of shapes of both the seam and the area which it outlines in the dorsal blastoderm sector is shown in Figs. 9: the dorsal views of living embryos are shown on the left while shown on the right are the schematic images of shaping of the blastoderm area in the incision sector. As the wound healing has not yet completed and epiboly is delayed, the seam bends in the blastoderm plane, its lateral points converging towards the centre of an area between the seam and blastoderm circumference. Moreover, the ER splits along the lines running from the lateral points of the seam to the blastoderm circumference just along the circumference radii. This completely outlines the lateral boundaries of the area under consideration whose intersection points with the blastoderm circumference converge to the sagittal plane (see Figs. 9). Shaping of this area, shown in Fig. 7D-F, perfectly corresponds to the ESH formation algorithm considered in the previous section. As a matter of fact, the dorsal sector shaping is subject to mass cell flows, the point of their convergence (intersection) initiating the ESH.

The wound area in the ventral incision sector is outlined in a similar way (data not shown), with the exception that its lateral boundaries diverge from the sagittal plane unless the wound healing is not completed. Then, only with the progress of epiboly, they move towards the sagittal plane, this being connected with decreasing the blastoderm circumference (with "waistline" formation). This differs from dorsal convergence in that the orientation of cell flows never deviates from the blastoderm longitudes at a given latitudinal diameter of the blastoderm circumference.

Ventral cutting causes a reversion of the dorsoventral axis, with embryonic shield formation in the initially ventral embryonic ring sector

We have shown that the removal of ventral blastoderm fragments in zebrafish embryos diverts the DV axis, a new ESH developing at the wound, that is, at the initially ventral blastoderm pole (Cherdantseva and Cherdantsev, 1985). In zebrafish this occurs if and only if, the operation is made before the onset of epiboly, at a stage shown in Fig 3. The reason for re-determination is simply that the healing wound creates a new centre of convergence both of tensile stresses and mass cell flows.

When we observed and measured the loach embryos after the ventral incisions made during ER formation, we found that sometimes the incision region manifests the behavior which one could have expected at the dorsal side. This occurred when the blastoderm circumference approximated the egg equator and lateral boundaries of incision area converged to the sagittal plane with no change in the blastoderm latitudinal diameter. Observation revealed that initial ESH vanished, as the ESH developed in the cut region.

Then, to make this reversion a reproducible event, we modified the cutting as shown in Fig. 10A (V-cuttings). The transversal ventral incision was supplemented by two lateral incisions running from the transversal incision lateral margins to the blastoderm circumference along the lines that deviated from the blastoderm longitudes so as to converge at the blastoderm margin. Wound healing orients cell flows (see Fig. 10B) so as to provide an opportunity of the new convergence centre originating in the ventral ER sector. In the control series the same operation was made in $\mathrm{ESH}$ region (D-cuttings).
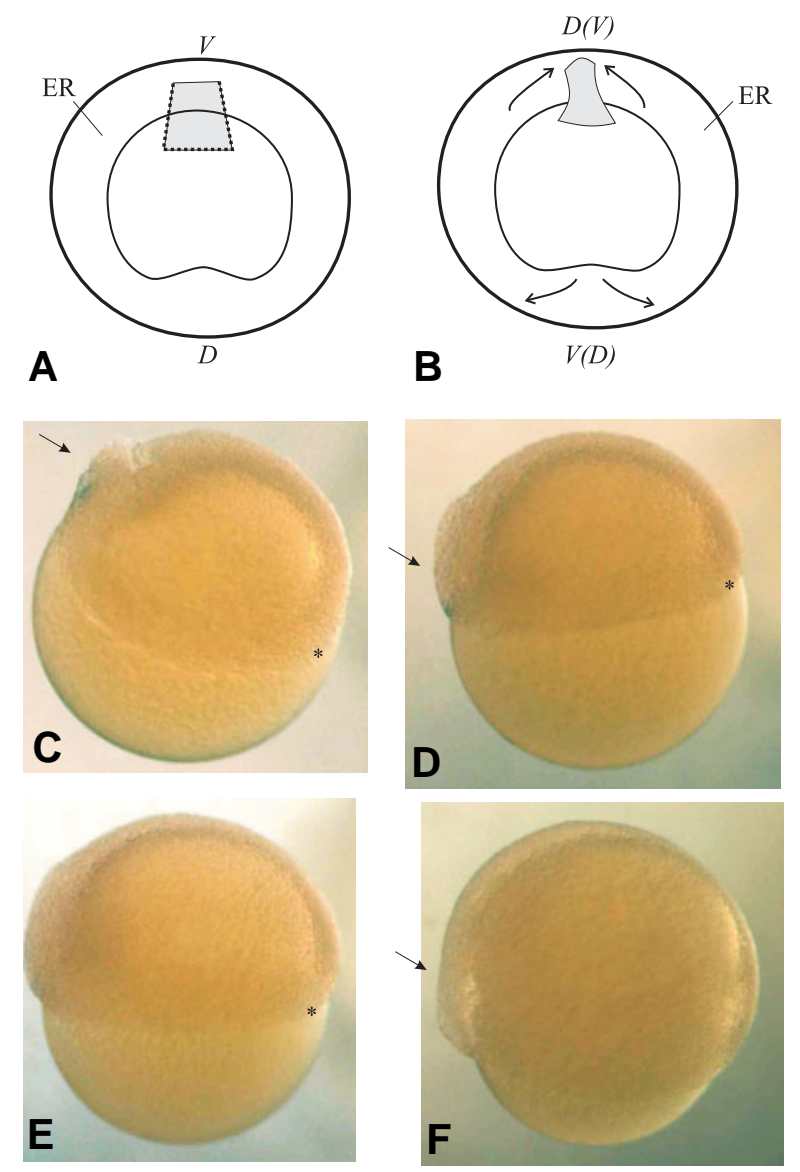

Fig. 10. Reversion of the DV axis by the ventral cutting. (A) Operation scheme; the dotted lines represent incisions; the shading represents the blastoderm in the cut region. (B) Scheme of the rearrangement of cell flows (arrows); $D(V)$, dorsalization of the initially ventral side; V(D), ventralization of the initially dorsal side of the embryo. (C-F) Successive steps of the formation of the new embryonic shield (ESH) in the living embryo. Asterisks, the initial ESH; arrows, the initially ventral side. 
A

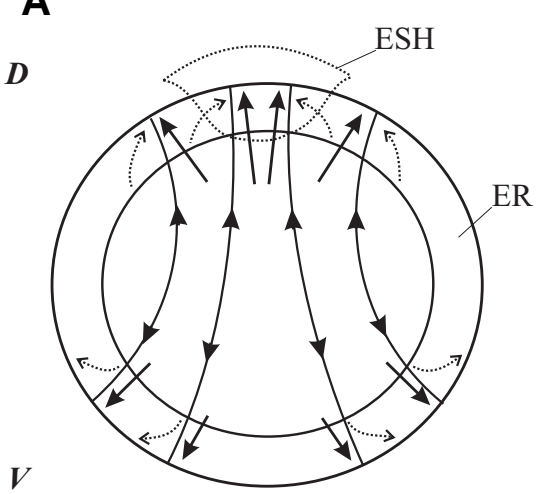

B

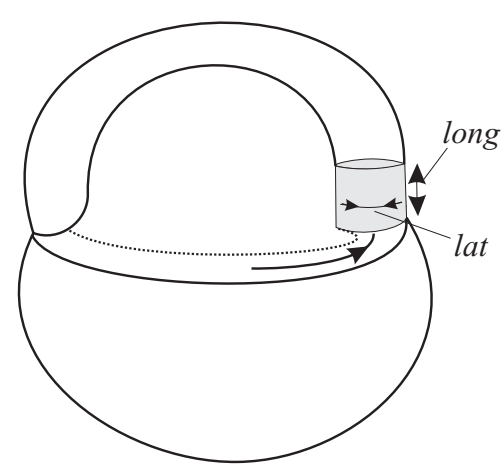

Fig. 11. Scheme of the formation of the DV axis in Teleost embryos. (A) The pattern of the tensile stresses and mass cell movements; black arrows, the centrifugal cell flows; light arrows, the convergence of the cell flows outlining the boundaries of the ESH ldotted contour). (B) Shaping of the embryo to counterbalance the preponderance of the latitudinal stresses at the dorsal side. the ESH is portrayed as a shaded cylinder. long, longitudinal; lat, latitudinal stresses. For other explanations, see text.

The results of these experiments are summarized in Table 1. A proportion of the operated embryos had an abnormal axial pattern and so it was impossible to trace the origin (dorsal or ventral) of axial structures without marking techniques. However, it was easy to do this in those cases in which the axial structures developed just as in the normal development, in the initial dorsal half, or at the opposite blastoderm side. As the cut traces (seams) are well-marked in living embryos, development could be followed for 12 hours after the operation without additional marks, provided that we aimed not to detect the precise ESH position, but only to find out if it developed in the initially dorsal or ventral blastoderm half.

The first signs of reversion become visible when the blastoderm circumference is to cross the egg equator (Figs. 10C, D). The operated ER sector becomes subject to thickening that exceeds that of the initial ESH situated at the opposite ER pole. After the blastoderm circumference crosses the equator, the initial ESH vanishes, its remnant being only at the very blastoderm edge, while the new ESH acquires the typical elongated shape of a well-formed ESH (Fig. 10D, E). If there were no trace of the wound, one could not have guessed that this ESH develops opposite to its normal location (Fig. 10F).

Thus the induction, by means of simple cutting, of a new centre of convergence of tensile stresses which, in turn, induces the formation of a new centre of convergence of cell flows, is sufficient to reverse the direction of the DV axis even at the ER formation stage. It follows that the origin of DV polarity can be by selforganization of mass cell movements on scale of the blastoderm circumference.

\section{Conclusions}

\section{The origin and maintenance of primary embryonic axes in the} early morphogenesis of Teleost embryos

It follows from our data that the primary embryonic axes are consequences of mass cell movements, rather than vice versa (see, for example, Driever, 1995). The general principle is that of movement-shaping equivalence, which means that the spatial series of differently shaped areas corresponds to the time succes-

sion of shaping of the same region (for details see Cherdantsev, 2003). The AP axis of the embryo is no more nor less than the longitudinal movement vector. It is subject to the principle that the blastoderm circumference is a matter of latitudinal contraction as it travels from one to another latitude of the egg surface. The DV axis, before the onset of epiboly, is a vector of cells pulled to one of the blastoderm halves in order to equilibrate the difference in the surface tensions at opposite blastoderm margins at their separation boundaries with the extra-embryonic structures.

After ER formation the relationship between the AP and DV axes is more complicated. Due to the convergence of mass cell flows, the interactions between the AP and DV movement-shaping vectors in the ER region become those of ESH formation. This interaction is again subject to the movement-shaping equivalence principle as it is the proportion between longitudinal stretching and latitudinal contraction of a moving area that is subject to change as the region moves over the outer embryonic surface. Meanwhile, one cannot say that at any outer surface point the convergence is simply a composition of longitudinal (AP) and latitudinal (DV) vectors in any coordinate system, if only because shaping the region when it changes its latitudinal-to-longitudinal position implies change in its movement trajectory (see the movement algorithm shown in 7D-F).

It follows that the orthogonal orientation of AP and DV axes requires explanation not in Cartesian, but rather in morphodynamics terms. If convergence of tensile stresses and mass cell movement trajectories had not been shown to be a matter of fact, they could have been deduced, by matching the orthogonal orientation of the $A P$ and DV axes to the shape changes at the opposite blastoderm margins in the course of epiboly. At the dorsal side of the embryo the amplitude of the bulging-flattening cycle is higher than at the ventral one. Meanwhile, both the dorsal and ventral margins move over the egg surface at equal rates (see Figs. 5 and 6). The mechanical equilibrium principle requires that the higher bulgingflattening scales of the shaping of the dorsal blastoderm margin be equilibrated by concentrating tensile stresses (the cell net) at the separation boundary between this margin and $Y C$. This is the origin of the convergence of tensile stresses, shown in Fig. 11 A by lines with arrowheads. This figure includes a schematic drawing of the blastoderm cross-section perpendicular to the AP axis across the

TABLE 1

\section{THE LONG-TERM RESULTS OF CUTTING EXPERIMENTS}

\begin{tabular}{lcccc} 
Operation & $\begin{array}{c}\text { Number of } \\
\text { embryos }\end{array}$ & \multicolumn{3}{c}{ Results(\%) } \\
\cline { 3 - 5 } & Reg & Trans & Abn \\
\hline V-cutting & 18 & 12 & 66 & 22 \\
D-cutting & 24 & 66 & 0 & 34 \\
\hline
\end{tabular}

Reg-regulation (axial structures develop in the initially dorsal half, Trans - Transdetermination (axia structures develop in the initially ventral half), Abn anormal development of axial structures. The differences between the experimental (V-cutting) and control (D-cutting) series are statistically significant at $p$ $<0.01$ 
blastoderm circumference. Bollard's centrifugal cell flows (which are preponderant on the dorsal side) are shown by black arrows, while the lighter arrows show their convergence triggering ESH formation when the blastoderm circumference approximates the equatorial region.

To explain why this makes the main embryonic axes maintain orthogonal orientation, recall that in the course of epiboly each new latitude which is to be embraced by the blastoderm circumference is subject to decrease so as to equalize its new circumferential diameter with the previous one. It follows that with ER formation ER molds its movement as if were on the cylindrical, rather than spherical surface, as shown in Fig. 11B. The latitudinal-to-longitudinal ratio of tensile stresses in a cylindrical shell is proportional to the shell thickness (Landau and Livshits, 1960). Therefore, as in the dorsal ER sector the blastoderm thickness is greater than in the ventral one, at the dorsal side there is an excess of latitudinal stresses which are equilibrated by the convergence of tensile stresses increasing the proportion of the blastoderm longitudinal stretching. The convergence acts like a geometric rigidity rib which turns into substantial rigidity after ESH formation, shown in Fig. $11 \mathrm{~B}$ as a shaded cylinder. Thus, from the mechanical point of view, $\mathrm{ESH}$ formation is the price for maintaining the orthogonal orientation of the main embryonic axes.

\section{Self-organization and prepattern}

As DV axis formation can be restarted in embryos having ER, provided that inside the ER domain there arises an opportunity of initiating a new centre of converging the mass cell movements tracks, the ESH can originate on the basis of random fluctuations, that is, on the basis of self-organization which ignores the ER prepattern. In fact, the morphodynamics algorithm of ESH formation shown in Figs. 7D-F contains all that is necessary for selforganization.

Imagine that the ER is uniform along its circumference along which, for arbitrary causes, there arises an infinitely small centre of cell movement trajectory convergence. The greater is its degree, the greater is the latitudinal contraction of the blastoderm circumference, this causing cell movement tracks to further converge. Thus, between the geometry and dynamics of an arbitrarily chosen ER sector there arises a positive feedback that enhances a small random fluctuation in only one direction, that of convergence. On the other hand, as any convergence centre recruits neighboring cells into its own movement-shaping, the probability of originating centers in neighboring places decreases, if only because the surrounding areas are depopulated. If this negative feedback has a spatial range of the whole ER, then, as it followed from our experiments on the reversing DV axis, the self-organization scenario guaranties that the ESH will develop as a single copy.

Even the possibility of such a scenario makes it necessary to reject a straightforward causal relationship in the time succession of morphogenetic events. Note that the converging lines of tensile stresses in Fig. 11A are shown normalized with respect to the blastoderm circumference. It follows that this convergence should not be confounded with that arising in the ESH formation process and based on a positive feedback between the deviation of tensile stresses from normal orientation and that of cell movement tracks. The convergence of tensile stresses, which is shown in Fig. 11A, is only a prepattern for ESH formation, whose morphogenetic significance consists in promoting self-enhancement of random deviations of cell movement tracks from normal orientation in the dorsal sector and preventing them at the opposite side of the blastoderm. The anisotropy of tensile stresses is known to promote intercalation of cells ("tensotaxis", see Beloussov et. al., 2000) triggering ESH formation in the dorsal ER sectors.

It follows that, while in developmental succession a prepattern precedes the formation of morphogenetic patterns, the reverse seems to be true both in the logical and evolutionary sense. In general mathematical terms, a prepattern offers parameters for the mass cell movement equations, only provided that the equations are those of self-organizing morphodynamics patterns (Beloussov, 1998; Cherdantsev, 2003).

One can outline a hypothetical evolutionary succession of steps at which the earlier and later morphogenetic events come into causal relationships that are rather parametric than causal ones. Imagine that in the beginning of morphogenetic evolution there is no prepattern at all, the blastoderm circumference being spatially homogeneous. The egg equator is a natural geometric singularity of mapping the plane into the plane (Whitney, 1955). This provides an opportunity to initiate the convergence both of tensile stresses and cell movement tracks, the attempt to make a "waistline" at the equatorial plane. We assume that this proceeds as in the cutting experiments with DV polarity reversion, with that exception that there is no initial ESH site. It follows that the convergence centers series can arise at random, their competition leaving only one copy for further development. This scenario is not so hypothetical as it could have seemed, because in some egg clutches, particularly in those consisting of small eggs, the formation both of ER and ESH is retarded until the blastoderm circumference crosses the egg equator (our unpublished observations).

Thus, at the first hypothetical step, the place of initiating the ESH has zero inheritance and information value. However, the accumulation of differently directed selection vectors operating on fitness with no functional relation to morphogenesis (see Cherdantsev et. al., 1996) may shift the convergence of tensile stresses to the very onset of epiboly (this may be due to a general increase in the contractile activity of the cell blastoderm network) and so there arises a tensile pattern shown in Figs. 11A. The choice of convergence site can be arbitrary, but, as it is imprinted in the ESH position, it acquires an information value, which is not be confounded with that in positional information theory (cf. Wolpert, 1996) and non-zero inheritance, as in different Teleost species the convergence of the tensile stresses can rest on different inherited characters of the embryo. The next step is the translation of DV polarity to the blastoderm with a flat blastoderm-YC separation boundary, as shown in Fig. 3 and so forth, up to imposing DV polarity on the fertilized egg. Thus, the beginning of development from the egg with DV polarity is in the end of the outlined evolutionary scenario.

\section{Acknowledgements \\ This study was supported by the Russian Fund of Fundamental Research, grant No. 0504-488479.}

\section{References}

ARNOLS W. G. A., van den BIGGELAAR J. A. M., VERDONK N. H. (1983). Spatial aspects of cell interactions involved in the determination of dorsoventral polarity in equally cleaving Gastrpods and regulative abilities of their embryos, as 
studied by micromere deletions in Lymnaea and Patella. W. Roux'Arch. Dev. Biol. 192: $75-85$.

BALLARD W. W. (1966). A new fate map for Salmo gairdneri. J. Exp. Zool., 184: 4973.

BALLARD W. W. (1981). Morphogenetic movements and fate maps of vertebrates. Am. Zool. 21: 391 - 399.

BELOUSSOV L.V. (1998). The dynamic architecture of a developing organism. Kluwer Acad. Publishers.

BELOUSSOV L. V., LOUCHISKAIA N. N., STEIN A. A. (2000). Tension-dependent cell movements in the early gastrula ectoderm of Xenopus laevisembryos. Dev. Genes Evol. 210: 92-104.

BETCHKU T. and TRINKAUS J. P. (1978). Contact relations, surface activity and cortical microfilaments of marginal cells of the enveloping layer and of the yolk syncitial and yolk cytoplasmic layers of Fundulus before and during epiboly. J. Exp. Zool. 206: $381-426$.

CHERDANTSEV V.G. (2003). Morphogenesis and evolution. M KMK Ltd Press, (In Russian).

CHERDANTSEV V. G., KRESLAVSKY A. G., SEVERTSOV A. S. (1996). Episelective evolution. (van VALEN Ed.). Evolutionary Theory 11: $69-87$.

CHERDANTSEV V. G. and KRAUS Y.U. A. (1996). Gastrulation in the marine hydroid Dynamena pumila: An example of evolutionary anticipation based on developmental self-organization. (van Valen Ed.). Evolutionary Theory 11: 89 $-98$.

CHERDANTSEV V. G. and TSVETKOVA N. V. (2005). Dynamics and variability of the early morphogenesis in loach (Misgurnus fossilis L.) based on observations of individual developmental paths. Ontogenez (Russ. Journ. Devel. Biol.) 36: 211-221

CHERDANTSEVA E. M. and CHERDANTSEV V. G. (1985). Determination of the dorsoventral polarity in a Teleost, Brachidanio rerio. Ontogenez (Sov. Journ. Dev. Biol.) 16: 270-280 (In Russian).

DRIVER W. (1995). Axis formation in zebra-fish. Curr. Opin. Dev. 5: 610-618.

GORDON, R. (1999). The Hierarchical Genome and Differentiation Waves: Novel Unification of Development, Genetics and Evolution. World Scientific \& Imperial College Press, Singapore \& London.

GORDON, R., BJÖRKLUND, N.K. and NIEUWKOOP, P.D. (1994). Dialogue on embryonic induction and differentiation waves. Int. Rev. Cytol. 150: 373-420.

KIMMEL C. B., WARGA R. M., SHILLING T. F. (1990). Origin and organization of the zebrafish fate map. Development 108: 581 - 595.

KRAUS Ju. A. and CHERDANTSEV V. G. (1999). Variability and equifinality of early morphogenesis in a marine hydroid, Dynamena pumila L. Ontogenez (Russ. Journ. Devel. Biol.) 28: 119 - 129.

NEKLIUDOVA I. V., KORWIN-PAVLOVSKAIA E. A., CHERDANTSEV V. G. (2003). Dorsoventral differences in morphogenetic potencies of the loach blastoderm in its fragments explantation experiments. 2001. Ontogenez (Russ. Journ. Dev. Biol.) 30: 353-361.

B. SCHMITZ and J. CAMPOS -ORTEGA J. (1994). Dorsoventral polarity of the zebrafish embryo is distinguishable prior to the onset of gastrulation. W. Roux' Arch. Dev. Biol. 203: 374-380.

WADDINGTON C. H. (1964). New pattern in development and genetics. Chicago Univ. Press.

WEISMANN A. (1893). The germ-plasm: A Theory of Heredity. London: Walter Scott.

WHITNEY.H. (1955). Mappings of the plane into the plane. Ann. Math.62: 374-410.

WOLPERT L. (1996). One hundred years of positional information. Trends in Genetics 12: 359-364. 